\title{
A reduced graphene oxide-based fluorescence resonance energy transfer sensor for highly sensitive detection of matrix metalloproteinase 2
}

This article was published in the following Dove Press journal:

International Journal of Nanomedicine

15 April 2016

Number of times this article has been viewed

\author{
Gaina $\mathrm{Xi}^{\prime}$ \\ Xiaoping Wang ${ }^{2}$ \\ Tongsheng Chen' \\ 'MOE Key Laboratory of Laser Life \\ Science \& College of Biophotonics, \\ South China Normal University, \\ ${ }^{2}$ Department of Pain Management, \\ The First Affiliated Hospital of Jinan \\ University, Guangzhou, People's \\ Republic of China
}

\begin{abstract}
A novel fluorescence nanoprobe (reduced nano-graphene oxide [nrGO]/fluorescein isothiocyanate-labeled peptide [Pep-FITC]) for ultrasensitive detection of matrix metalloproteinase 2 (MMP2) has been developed by engineering the Pep-FITC comprising the specific MMP2 substrate domain (PLGVR) onto the surface of nrGO particles through non-covalent linkage. The nrGO was obtained by water bathing nano-graphene oxide under $90^{\circ} \mathrm{C}$ for 4 hours. After mixing the nrGO and Pep-FITC for 30 seconds, the fluorescence from Pep-FITC was almost completely quenched due to the fluorescence resonance energy transfer between fluorescein isothiocyanate (FITC) and nrGO. Upon cleavage of the amide bond between Leu and Gly in the Pep-FITC by protease-MMP2, the FITC bound to nrGO was separated from nrGO surface, disrupting the fluorescence resonance energy transfer process and resulting in fluorescence recovery of FITC. Under optimal conditions, the fluorescence recovery of nrGO/Pep-FITC was found to be directly proportional to the concentration of MMP2 within $0.02-0.1 \mathrm{nM}$. The detection limit of the nrGO/Pep-FITC was determined to be $3 \mathrm{pM}$, which is approximately tenfold lower than that of the unreduced carboxylated nano-graphene oxide/Pep-FITC probe.
\end{abstract}

Keywords: graphene oxide, reduction, high sensitive detection, fluorescence

\section{Introduction}

Matrix metalloproteinases (MMPs) are a family of zinc-dependent endoproteases capable of degrading virtually all kinds of extracellular matrix and basement membrane components. ${ }^{1}$ MMPs target substrates, including proteinases, proteinase inhibitors, clotting factors, cell-cell adhesion molecules, chemotactic molecules, latent growth factors, growth factor-binding proteins, and cell surface receptors. ${ }^{2}$ MMPs were also found to interfere with the induction of apoptosis in malignant cells, especially after chemotherapy, by cleaving Fas ligand from the surface of cancer cells with MMP7. ${ }^{3}$ Tumors are highly vascularized tissues, and the formation of new blood vessels can be triggered by the release of vascular endothelial growth factor that is mainly facilitated by MMP2 and MMP9. ${ }^{4}$ In addition, degradation of extracellular matrix components and other extracellular molecules by MMPs may generate fragments with new bioactivities inhibiting angiogenesis. ${ }^{5}$ For example, it was reported that degradation of plasminogen by MMP2, MMP9, and MMP12 produced significant amounts of angiostatin, a cleavage product with antiangiogenic function. ${ }^{6,7}$

Sensitive, rapid, and accurate detection of MMPs is of great significance for clinical disease diagnosis, therapy, and MMP inhibitors screening. High levels of MMPs have been observed in the serum/blood, ${ }^{8,9}$ saliva, ${ }^{10}$ and urine ${ }^{11}$ of patients with 
cancers, and in the plasma of patients with diabetes ${ }^{12}$ or hypertensions. ${ }^{13}$ Gelatin zymography is a qualitative MMPs detection method. ${ }^{14}$ Enzyme-linked immunosorbent assay, a conventional and most widely used method in clinical assay, is very sensitive, but the antibody proteins are expensive and difficult to be acquired. In addition, enzyme-linked immunosorbent assay method needs tedious separation operations with at least 5 hours to detect the activity of MMPs. ${ }^{15}$ Fluorescence resonance energy transfer (FRET) refers to the energy transfer between donor and acceptor fluorophores within 1-10 nm distance range, and the rate of this long-range resonance energy transfer was suggested to have a (distance) ${ }^{-6}$ dependence. ${ }^{16}$ Therefore, many FRETbased sensors have been developed for sensitive and rapid detection of MMPs ${ }^{17,18}$ and other trace molecules. ${ }^{19}$

Graphene oxide (GO), a novel one-atom-thick twodimensional carbon material, has been demonstrated to be a remarkable FRET acceptor for various fluorescent dyes owing to the excellent ability for absorbing visible light. ${ }^{19-22}$ Many kinds of GO-based FRET sensors have been developed for sensitive MMP2 detection. ${ }^{23-25}$ Reduced graphene oxide (rGO) is a more effective quencher due to the higher ability to absorb visible light over GO. ${ }^{26-28}$ There are many ways to prepare rGO by using strong alkaline, alcohols, natural extracts, or overheated supercritical water in sealed container as reductant. ${ }^{29-32}$ We recently bathed covalently PEGylated nano-graphene oxide (nGO-PEG, here "PEG" means polyethylene glycol) in pure water under $90^{\circ} \mathrm{C}$ for 24 hours to obtain the reduced nGO-PEG that had an $\sim 7$.6-fold increment in near-infrared (NIR) absorption at $808 \mathrm{~nm}$ over $\mathrm{nGO}-\mathrm{PEG}$ and was stable for $>3$ months under $4^{\circ} \mathrm{C} .^{33,34}$

In this study, we developed a reduced nano-graphene oxide (nrGO)-based FRET sensor (nrGO/fluorescein isothiocyanate-labeled peptide [Pep-FITC]) for highly sensitive MMP2 detection. As illustrated in Figure 1, the functionalized nrGO/Pep-FITC sensor was prepared by non-covalent binding of Pep-FITC ( $\mathrm{H}_{2} \mathrm{~N}$-PEG-CALNNGPLGVRGRAKFITC) containing the core cleavage substrate sequence (PLGVR) of MMP2 25 to nrGO that was reduced by bathing carboxylated nano-graphene oxide (c-nGO) in water at $90^{\circ} \mathrm{C}$ for 4 hours. The nrGO/Pep-FITC sensor was able to quantitatively detect the concentration of MMP2 in the range of $0.02-0.1 \mathrm{nM}$. The detection limit of the $\mathrm{nrGO} /$ Pep-FITC was determined to be $3 \mathrm{pM}$, which is approximately tenfold lower than that of the $\mathrm{c}-\mathrm{nGO} / \mathrm{Pep}-\mathrm{FITC}$ probe. To the best of our knowledge, this is the first report on an nrGO-based biosensing platform for analyzing MMP2.

\section{Materials and methods}

\section{Chemicals and materials}

Pep-FITC ( ${ }_{2}$ N-PEG-CALNNGPLGVRGRAK-FITC) was synthesized by Shanghai ABBiochem Co, Ltd (Shanghai, People's Republic of China). Graphite powder was purchased from Nanjing XFNano Materials Tech Co, Ltd (Nanjing, People's Republic of China). Latent proMMP2 was purchased from Sino Biological Inc Co, Ltd (Beijing, People's Republic of China). 4-Aminophenylmercuric acetate (APMA), 1,10-phenanthroline, and dimethyl sulfoxide were purchased from Sigma-Aldrich (St Louis, MO, USA). Other reagents employed were all of analytical grade, and were used without further purification. All the buffers were prepared with tri-distilled water, which was purified with a water purification system (ELGA; High Wycombe, Bucks, UK) to a specific resistance of $18.2 \mathrm{M} \Omega \cdot \mathrm{cm}$.

\section{Synthesis of c-nGO and nrGO}

GO was prepared by a modified Hummer's method utilizing expandable graphite flake. ${ }^{35,36} \mathrm{GO}$ was sonicated with Ultrasonic Cell Crusher (JY92-2D; Xinzhi Ultrasonic Equipment Co, Ningbo, People's Republic of China) for 1.5 hours to give a clear solution in an ice bath to obtain nano-graphene oxide (nGO) suspension. c-nGO was obtained by the reaction with $\mathrm{NaOH}(1.2 \mathrm{~g})$ and $\mathrm{ClCH}_{2} \mathrm{COOH}(1 \mathrm{~g})$ for 0.5 hour following the known procedure. ${ }^{37}$

c-nGO solution $(\sim 1 \mathrm{mg} / \mathrm{mL})$ was transferred to a sealed glass bottle and then bathed at $90^{\circ} \mathrm{C}$ for 4 or 12 hours to get nrGO (nrGO [4 h] or nrGO [12 h]).

\section{Characterizations}

The hydrodynamic diameters and $\zeta$-potentials of nGO, c-nGO, nrGO (4 h), and nrGO (12 h) were measured by Zetasizer Nano ZS (Malvern Instruments Ltd, Malvern, UK). The materials were imaged with tapping mode atomic force microscope (OLTESPA probe, Bruker Dimension Icon; Bruker, Ettlingen, Germany) on a mica substrate. The images of all samples were recorded using a digital camera (Nikon, Tokyo, Japan) with 1,280×1,280 pixels resolution. Ultraviolet-visible (UV-vis) spectra were obtained by a UV-vis spectrometer (Lambda 35; PerkinElmer, Waltham, MA, USA) with a $1 \mathrm{~cm}$ quartz cuvette. Fourier transfer infrared (FTIR) spectra were recorded on a Nicolet 6700 FTIR spectrometer (Thermo Fisher Scientific, Waltham, MA, USA). Raman spectra were taken with a Renishaw (Wotton-under-Edge, UK) inVia micro-Raman spectroscopy system equipped with a $514.5 \mathrm{~nm} \mathrm{Ar}^{+}$laser. X-ray diffraction spectra were obtained using a D8 ADVANCE 

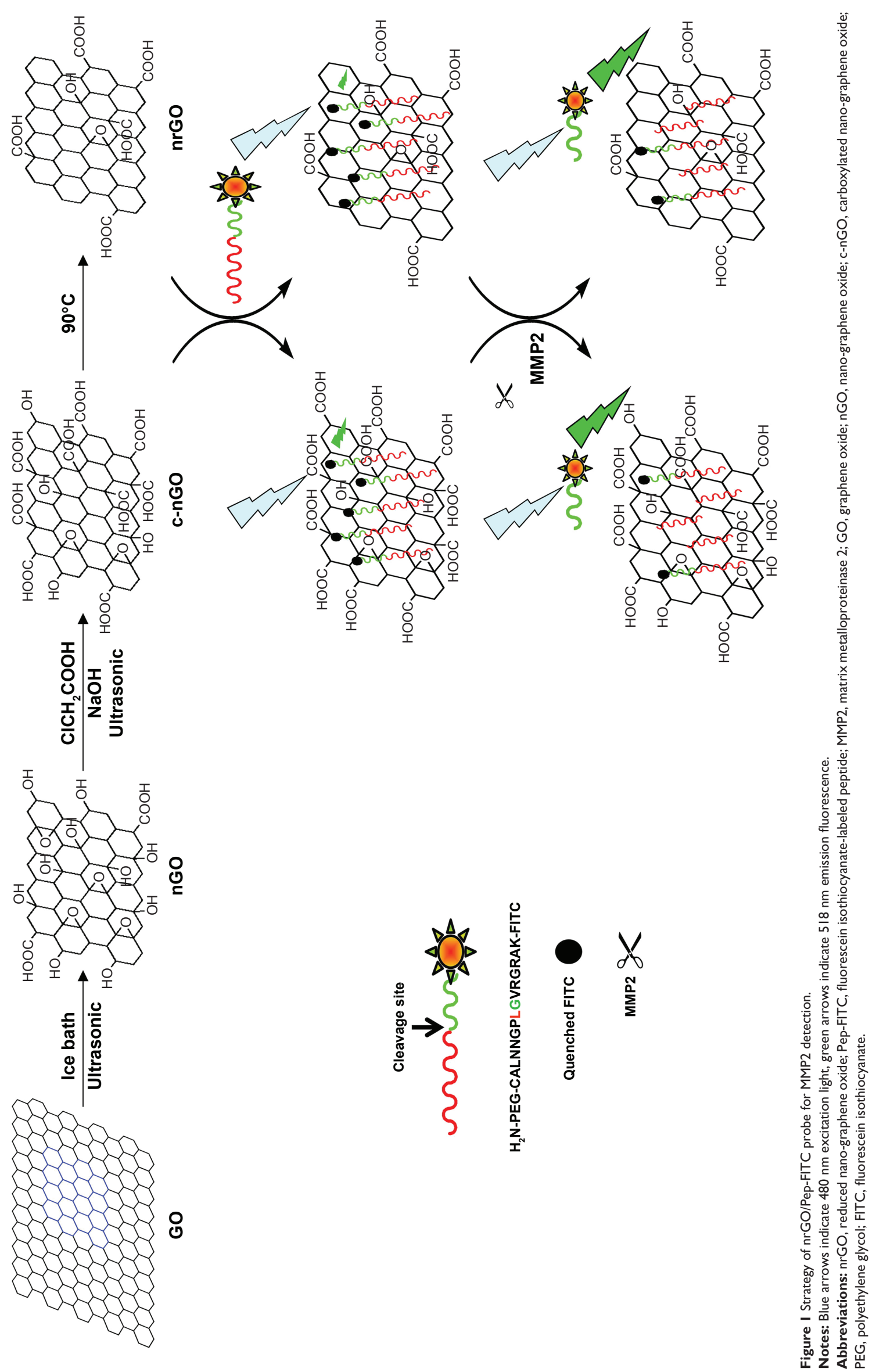
(Bruker) using $\mathrm{Cu} \mathrm{K} \alpha(1.5406 \AA)$ radiation. Luminescence Spectrometer (LS 55; PerkinElmer) was used to measure fluorescence emission. The excitation wavelength and emission wavelength were 480 and $518 \mathrm{~nm}$, respectively. Both the excitation and emission slits were set at $10 \mathrm{~nm}$.

\section{Preparation of c-nGO/Pep-FITC and nrGO/Pep-FITC sensors}

Unless otherwise noted, nrGO/Pep-FITC sensor was prepared by treating the Pep-FITC $(100 \mathrm{nM})$ with $\mathrm{nrGO}(1 \mu \mathrm{g} / \mathrm{mL})$ in the TCNB (50 mM Tris, $10 \mathrm{mM} \mathrm{CaCl}_{2}, 150 \mathrm{mM} \mathrm{NaCl}$, and $0.05 \%$ Brij 35; pH 7.5) buffer. c-nGO/Pep-FITC was prepared as described for the $\mathrm{nrGO} / \mathrm{Pep}-\mathrm{FITC}$.

\section{Detection of MMP2 with c-nGO/Pep- FITC and nrGO/Pep-FITC sensors}

Before detection, latent proMMP2 was first activated with APMA according to the known procedure. ${ }^{38} \mathrm{MMP} 2$ in TCNB buffer was added with $1 \mathrm{mM}$ APMA (final concentration), and the mixture was incubated in heating thermostat blender (LTH100; Shanghai LNB Instrument Co, Ltd, Shanghai, People's Republic of China) at $37^{\circ} \mathrm{C}$ for 1 hour with gentle shaking.

In an Eppendorf tube loaded with $100 \mathrm{nM}$ c-nGO/PepFITC or nrGO/Pep-FITC (final concentration), $1 \mu \mathrm{g} / \mathrm{mL}$ of activated MMP2 was added, and the mixtures were incubated at $37^{\circ} \mathrm{C}$ with gentle shaking for 2 hours, and fluorescence intensity of each sample was then recorded with $\lambda_{\mathrm{ex} / \mathrm{em}}=480 / 518 \mathrm{~nm}$.

\section{Results and discussion \\ Preparation and characterization of nrGO}

The hydrodynamic sizes of nGO, c-nGO, $\operatorname{nrGO}(4 \mathrm{~h})$, and nrGO (12 h) nanoparticles were firstly analyzed by dynamic light scattering (DLS). In order to assess the effect of bathing time on $\mathrm{GO}$ reduction degree, we bathed $\mathrm{GO}$ under $90^{\circ} \mathrm{C}$ for 4 and 12 hours. As shown in Figure 2A, the DLS hydrodynamic diameter of nGO, c-nGO, $\operatorname{nrGO}(4 \mathrm{~h})$, and $\mathrm{nrGO}(12 \mathrm{~h})$
A

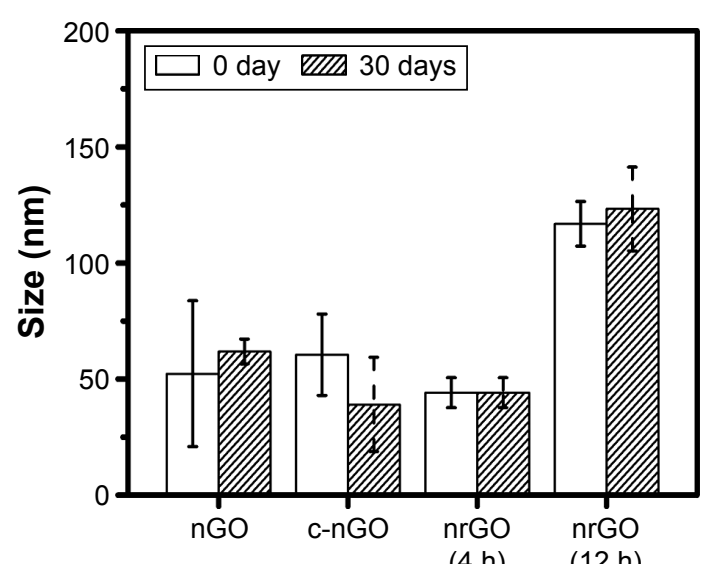

$(4 \mathrm{~h})$

C
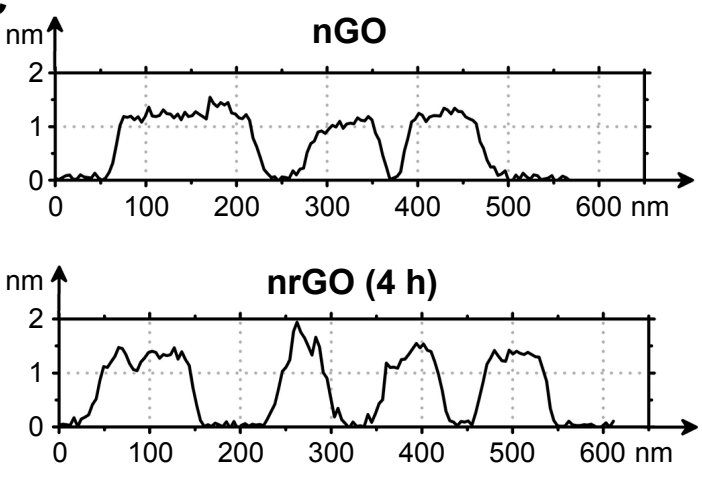

B
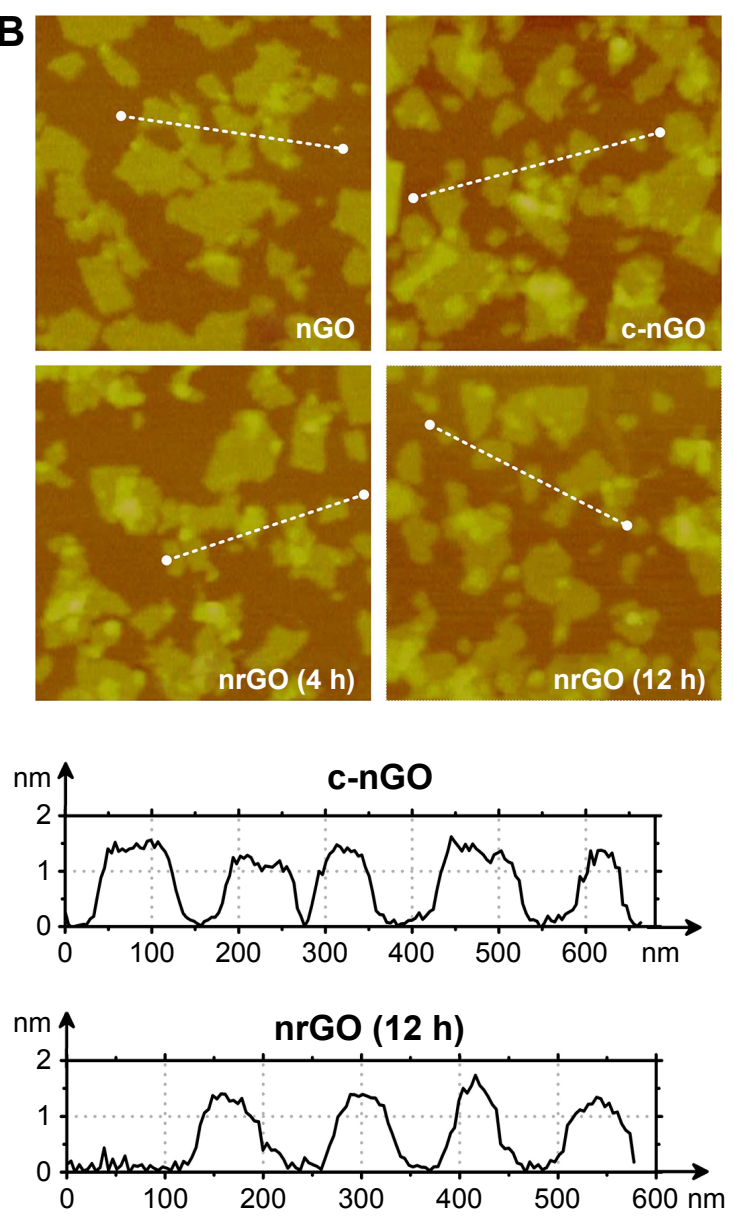

Figure 2 Size of nano-materials.

Notes: Hydrodynamic diameters (A), AFM images (B), Diameter and thickness (C) (data corresponds to the lines in figure [B]) of $\mathrm{nGO}$, c-nGO, nrGO (4 h), and nrGO $(12 \mathrm{~h})$. nrGO $(4 \mathrm{~h})$ and nrGO $(12 \mathrm{~h})$ were formed by bathing $\mathrm{nGO}$ under $90^{\circ} \mathrm{C}$ water for 4 and 12 hours, respectively. The materials were stored under $4{ }^{\circ} \mathrm{C}$.

Abbreviations: AFM, atomic force microscopy; nGO, nano-graphene oxide; c-nGO, carboxylated nano-graphene oxide; nrGO, reduced nano-graphene oxide; h, hours. 
was holding $\sim 50,50,50$, and $120 \mathrm{~nm}$, respectively, during 1 month. We next used atomic force microscopy (AFM) images to further measure the diameters of the nGO, c-nGO, nrGO (4 h), and nrGO (12 h) nanoparticles. AFM data showed sheets of $\sim 80 \mathrm{~nm}$ diameter (assuming that these are in round shape) for $\mathrm{nGO}, \mathrm{c}-\mathrm{nGO}, \mathrm{nrGO}(4 \mathrm{~h})$, and $\mathrm{nrGO}$ $(12 \mathrm{~h})$, and the sheet thickness of nGO, c-nGO, nrGO (4 h), and $\mathrm{nrGO}(12 \mathrm{~h}$ ) was $\sim 1.5 \mathrm{~nm}$ (Figure $2 \mathrm{~B}$ and $\mathrm{C}$ ). Difference between AFM size and DLS hydrodynamic size may be due to the sheet-like structures of GO and rGO.

It is well known that the edges of nGO or/and some parts of its basal plane are terminated by hydrogen, hydroxyl, carboxylic, and epoxy groups. ${ }^{39}$ Reduction reaction is unable to remove all oxygen groups of nGO, and the residual negatively charged oxygen functional groups allow nrGO solution to be dispersed well in water due to electrostatic repulsion mechanism, ${ }^{40,41}$ which may be the reason why the sizes of nrGO remain stable during 1 month at $4^{\circ} \mathrm{C}$ (Figure 2A). This view was further confirmed by the stable $\zeta$-potential values $(\sim-40 \mathrm{mV})$ of the $\mathrm{nGO}, \mathrm{c}-\mathrm{nGO}, \operatorname{nrGO}(4 \mathrm{~h})$, and $\mathrm{nrGO}$ (12 h) during 1 month (Figure $3 \mathrm{~A})$. The stable diameter and $\zeta$-potential demonstrated that the nGO, c-nGO, nrGO $(4 \mathrm{~h})$, and nrGO $(12 \mathrm{~h})$ solutions were very stable at $4^{\circ} \mathrm{C}$ during at least a month, which was also further verified by the stable images of nGO, c-nGO, $\operatorname{nrGO}(4 \mathrm{~h})$, and nrGO (12 h) (Figure 3B). The suspension remained clear under the same conditions. Moreover, the color of nGO, c-nGO, $\operatorname{nrGO}(4 \mathrm{~h})$, and $\operatorname{nrGO}(12 \mathrm{~h})$ solutions changed from brown to yellowish brown, dark brown, and black brown (Figure 3B), an evidence for the reduction of nGO. Furthermore, UV-vis spectrometer was used to detect the $\mathrm{UV}$-vis spectra of nGO, c-nGO, nrGO (4 h), and nrGO (12 h). As shown in Figure 3C, nGO had a strong optical absorption peak at $228 \mathrm{~nm}$ and a weak shoulder at $300 \mathrm{~nm}$. Compared with nGO, c-nGO exhibited higher absorbance at long wavelength $(>300 \mathrm{~nm})$, probably due to the partial and slight reduction of nGO under strong alkaline conditions. ${ }^{29}$ Compared with c-nGO, nrGO ( $4 \mathrm{~h}$ ) and nrGO (12 h) exhibited $\sim 4.29$ - and 6.88-fold increment in NIR absorption at $808 \mathrm{~nm}$ (Figure 3C), which further verified the degree of reduction. Moreover, the absorption peak $(226.5 \mathrm{~nm})$ of c-nGO corresponding to $\pi \rightarrow \pi^{*}$ transitions of aromatic $\mathrm{C}=\mathrm{C}$ bonds red-shifted to $\sim 235.5$ and $249.5 \mathrm{~nm}$ for $\operatorname{nrGO}(4 \mathrm{~h})$ and $\mathrm{nrGO}(12 \mathrm{~h})$, respectively, and the shoulder peak $(300 \mathrm{~nm})$ nearly disappeared for both $\mathrm{nrGO}(4 \mathrm{~h})$ and $\mathrm{nrGO}(12 \mathrm{~h})$, indicating partial restoration of the $\pi$-conjugation network of GO carbon structure and the effect of deoxygenation. ${ }^{42}$

FTIR spectroscopy revealed the existence of $\mathrm{OH}$ $\left(\sim 3,400 \mathrm{~cm}^{-1}\right), \mathrm{C}=\mathrm{O}\left(\sim 1,720 \mathrm{~cm}^{-1}\right), \mathrm{C}=\mathrm{C}\left(\sim 1,620 \mathrm{~cm}^{-1}\right)$,
$\mathrm{C}-\mathrm{OH}\left(\sim 1,380 \mathrm{~cm}^{-1}\right)$, and $\mathrm{C}-\mathrm{O}\left(\sim 1,060 \mathrm{~cm}^{-1}\right)$ functional groups in the nGO (Figure 3D), ${ }^{43}$ which indicated that the abundant oxygen-containing moieties were present on nGO surfaces. We activated nGO sample with chloroacetic acid under strongly basic conditions in order to activate epoxide and ester groups, and to convert hydroxyl groups to carboxylic acid moieties. ${ }^{44}$ This c-nGO having good water solubility and more carboxylic acid groups exhibited the characteristic peak of $\sim 1,600 \mathrm{~cm}^{-1}$ for carboxyl groups $\left(-\mathrm{COO}^{-}\right) .{ }^{25}$ After water-bathing treatment, the FTIR spectra of $\operatorname{nrGO}(4 \mathrm{~h})$ and nrGO (12h) were similar to that of c-nGO, indicating similar structure of the reduced graphene material with the c-nGO.

As shown in the X-ray diffraction patterns (Figure 3E), the $d$-spacing of sharp feature peak (001) was $1.01 \mathrm{~nm}$ (at $8.74^{\circ}$ ) for $\mathrm{nGO}, 0.89 \mathrm{~nm}\left(\right.$ at $9.84^{\circ}$ ) for c-nGO, $0.86 \mathrm{~nm}$ (at $10.25^{\circ}$ ) for $\operatorname{nrGO}(4 \mathrm{~h})$, and disappeared for $\operatorname{nrGO}(12 \mathrm{~h})$, which indicated that most of the oxygen functional groups, having marked effects on the $d$-spacing, had been reduced in this condition. The $d$-spacing of sharp feature peak (002) was $0.48 \mathrm{~nm}\left(\right.$ at $18.22^{\circ}$ ) for $\mathrm{nGO}, 0.39 \mathrm{~nm}\left(\right.$ at $22.46^{\circ}$ ) for c-nGO, $0.37 \mathrm{~nm}\left(\right.$ at $\left.23.40^{\circ}\right)$ for $\mathrm{nrGO}(4 \mathrm{~h})$, and $0.36 \mathrm{~nm}$ (at $24.38^{\circ}$ ) for nrGO $(12 \mathrm{~h})$, further confirming the gradual reduction of c-nGO with increasing water bath-treated times at $90^{\circ} \mathrm{C} .{ }^{45}$

Raman spectroscopy was used to characterize nGO, c-nGO, nrGO (4 h), or nrGO (12 h) (Figure 3F). The typical features in Raman spectra are the $G$ band at $1,600 \mathrm{~cm}^{-1}$ and the $\mathrm{D}$ band at $1,350 \mathrm{~cm}^{-1}$. The $\mathrm{G}$ band is usually assigned to the $\mathrm{E}_{2 \mathrm{~g}}$ phonon of $\mathrm{C} \mathrm{sp}^{2}$ atoms, while the prominent $\mathrm{D}$ band is an indication of disorder corresponding to defects associated with vacancies, grain boundaries, and amorphous carbon species. ${ }^{34}$ The intensity ratio $\left(I_{\mathrm{D}} / I_{\mathrm{G}}\right)$ of $\mathrm{D}$ band to $\mathrm{G}$ band was $\sim 0.812$ for $\mathrm{nGO}, 0.888$ for c-nGO, 0.849 for $\operatorname{nrGO}(4 \mathrm{~h})$, and 0.817 for $\operatorname{nrGO}(12 \mathrm{~h})$. Compared with nGO, c-nGO had larger $I_{\mathrm{D}} / I_{\mathrm{G}}$ value. However, the $I_{\mathrm{D}} / I_{\mathrm{G}}$ value of $\operatorname{nrGO}(4 \mathrm{~h})$ or nrGO (12 h) was lower than that of c-nGO, suggesting that the reduction reaction in this facile and green reduction strategy developed here was able to recover the aromatic structures by repairing defects.

\section{Preparation and optimization of $\mathrm{nrGO} /$ Pep-FITC sensors}

nrGO/Pep-FITC sensor was prepared by treating Pep-FITC $(100 \mathrm{nM})$ with nrGO $(1 \mu \mathrm{g} / \mathrm{mL})$ in TCNB buffer, and c-nGO/ Pep-FITC served as a control. The binding of biomolecules to $\mathrm{GO}$ is generally through $\pi-\pi$ and electrostatic interactions, ${ }^{46}$ and thus, the electrically charged nature of c-nGO and nrGO needs to be examined. As shown in Figure 3B, the $\zeta$-potential values of the c-nGO, $\operatorname{nrGO}(4 \mathrm{~h})$, and $\mathrm{nrGO}$ $(12 \mathrm{~h})$ were $\sim-40 \mathrm{mV}$, indicating that c-nGO, $\operatorname{nrGO}(4 \mathrm{~h})$, 


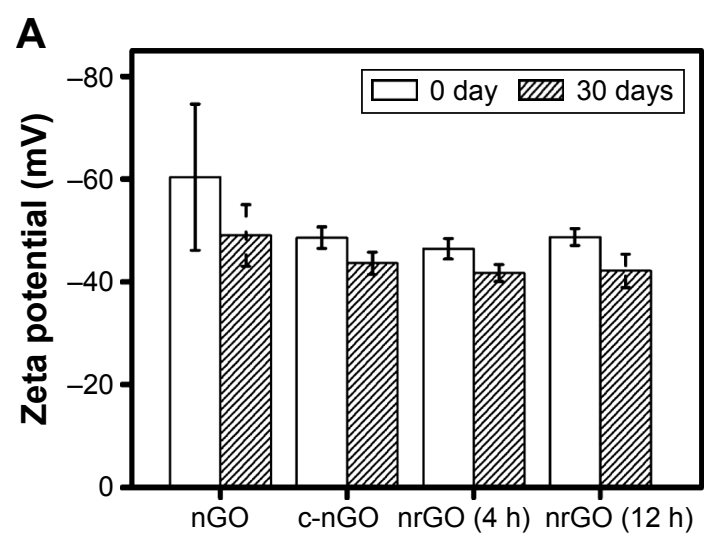

B
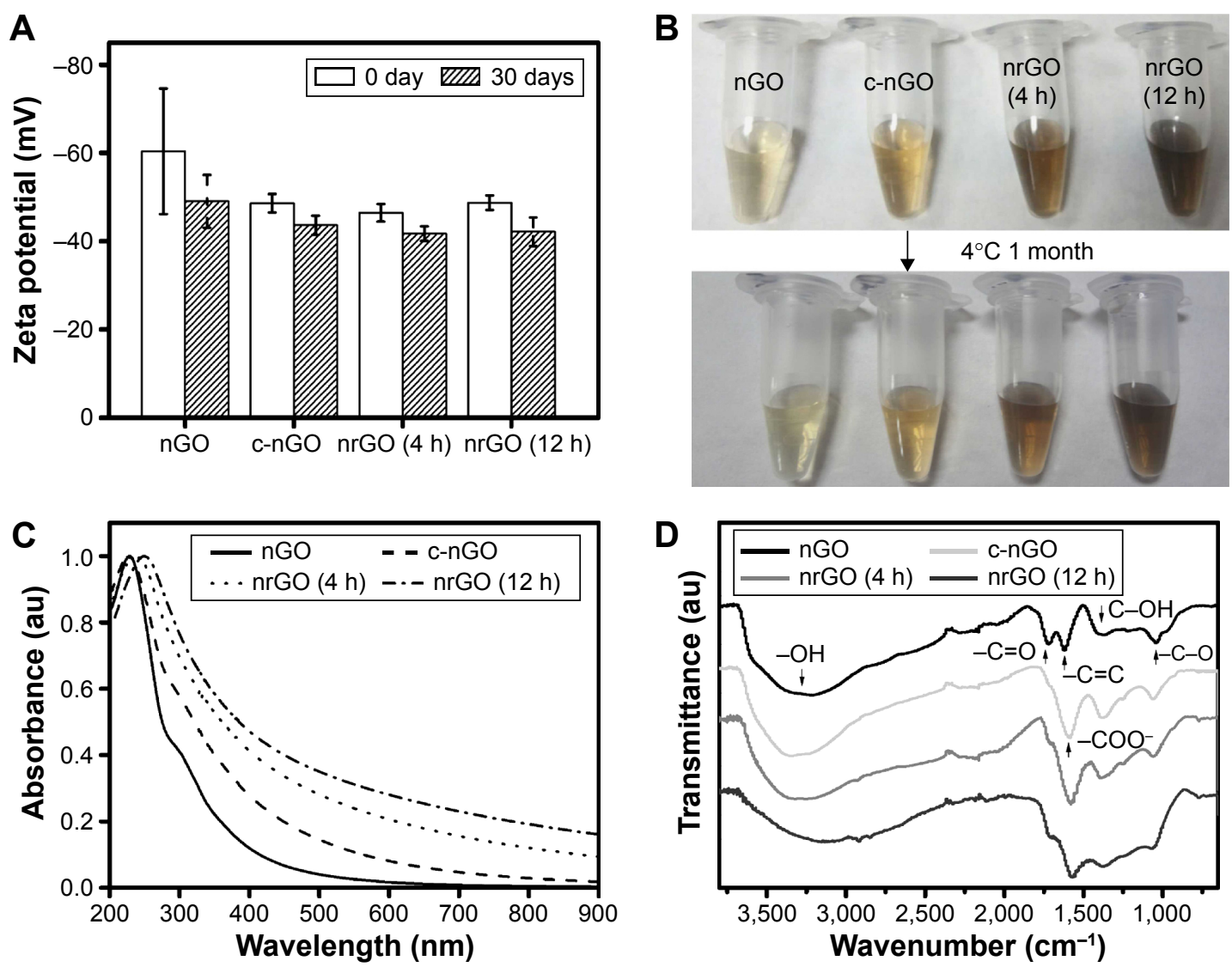

$\mathbf{E}$

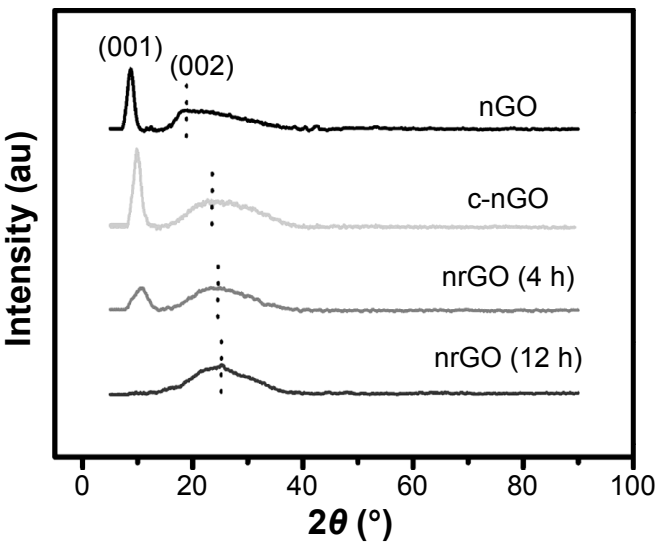

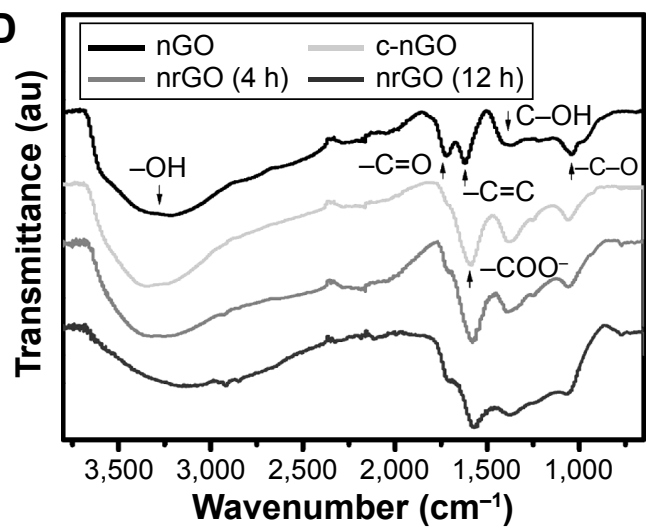

$\mathbf{F}$

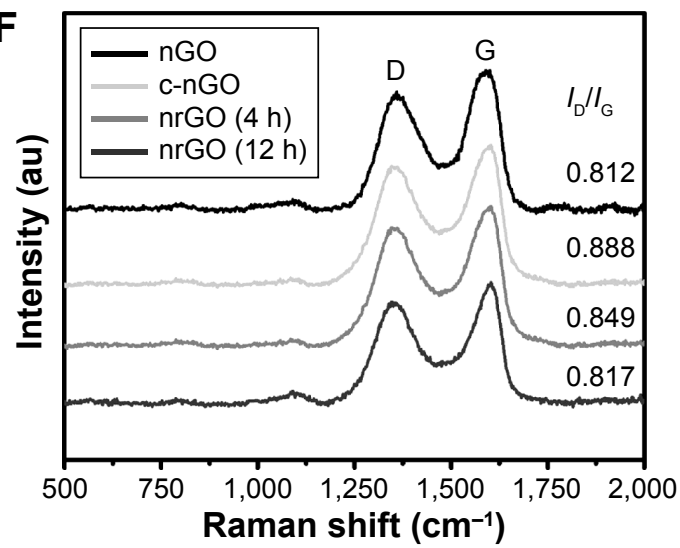

Figure 3 Characterizations of nano-materials.

Notes: Zeta potentials (A), photographs (B), UV-vis absorption spectra (C), FTIR spectra (D), XRD patterns (E), and Raman spectra (F) of nGO, c-nGO, nrGO (4 h), and $\operatorname{nrGO}(12 \mathrm{~h})$. nrGO $(4 \mathrm{~h})$ and nrGO $(12 \mathrm{~h})$ were formed by bathing $\mathrm{nGO}$ under $90^{\circ} \mathrm{C}$ water for 4 and $\mathrm{I} 2$ hours, respectively.

Abbreviations: FTIR, Fourier transform infrared; XRD, X-ray diffraction; nGO, nano-graphene oxide; c-nGO, carboxylated nano-graphene oxide; nrGO, reduced nanographene oxide; au, arbitrary unit; UV-vis, ultraviolet-visible; $I_{D} / I_{G}$, the intensity ratio of $D$ band to $G$ band in Raman spectra; h, hours.

and nrGO (12 h) were negatively charged. The pI value of our peptide calculated by ProtParam ${ }^{47}$ was 11.3 , so the peptide (Pep-FITC) would be positively charged at physiological $\mathrm{pH}$ of $\sim 7 .{ }^{24}$ In addition, the left side of the peptide chain was linked with PEG-NH${ }_{2}$, which was more conducive to the formation of the probes. In order to optimize the amount of nrGO or c-nGO as nanoquencher, different volumes of c-nGO, nrGO (4 h), or nrGO (12 h) solution $(0.1 \mathrm{mg} / \mathrm{mL})$ were mixed with Pep-FITC (100 nM) in TCNB buffer, and the fluorescence intensity of the solution was measured after incubation for 10 minutes. We found that the fluorescencequenching efficiency $\left(Q_{\mathrm{E}}[\%]=100 \times[1-\beta]\right.$, where $\beta$ is the ratio of fluorescence of the quenched-to-completely dequenched state) of Pep-FITC gradually increased along with the increasing concentration of c-nGO, nrGO (4 h), and $\operatorname{nrGO}(12 \mathrm{~h})$, and the $Q_{\mathrm{E}}$ value was $72.82 \%, 90.18 \%$, 
and $92.91 \%$ for $1 \mu \mathrm{g} / \mathrm{mL}$ of c-nGO, $\mathrm{nrGO}(4 \mathrm{~h})$, and $\mathrm{nrGO}$ $(12 \mathrm{~h}$ ), respectively (Figure $4 \mathrm{~A})$. The higher $Q_{\mathrm{E}}$ of $\mathrm{nrGO}$ over the unreduced c-nGO is due to the higher ability of nrGO to absorb visible light. Compared with c-nGO, nrGO showed a significant increment in NIR absorption (Figure 3C), leading to a bigger overlap area between the absorption spectrum of nrGO, the acceptor here, and the emission spectrum of the donor-FITC, and increasing the fluorescence-quenching efficiency of nrGO. Since there was no significant difference in the fluorescence-quenching ability between $\operatorname{nrGO}(12 \mathrm{~h})$ and nrGO (4 h) (Figure 4A), we chose nrGO (4 h) as nrGO for following test without indication.

We next investigated time dependence of the fluorescence quenching between nrGO and Pep-FITC. The fluorescence intensity $\left(\lambda_{\text {ex } / \mathrm{m}}=480 / 518 \mathrm{~nm}\right)$ of nanoprobes during preparation was monitored as a function of time using microplate reader (Infinite M200; Tecan, Männedorf, Switzerland). The fluorescence quenching of Pep-FITC by nrGO was very fast ( $<30$ seconds) after adding $\mathrm{nrGO}(1 \mathrm{mg} / \mathrm{mL})$ to the PepFITC solution $(100 \mathrm{nM})$, and the quenched fluorescence remained quite stable thereafter (Figure 4B), indicating that the binding equilibrium between $\mathrm{nrGO}$ and Pep-FITC was achieved immediately, ensuring a constant background for the subsequent MMP2 sensing.

\section{Detection of MMP2 using nrGO/Pep-FITC sensor}

We first examined the principle of nrGO/Pep-FITC for MMP2 detection. The fluorescence spectrum of c-nGO/ Pep-FITC sensors (100 nM) after adding the target MMP2, activated with APMA according to the known procedure, ${ }^{38}$ was obviously enhanced (Figure 5, curve b compared with curve a). However, the enhanced fluorescence spectrum of c-nGO/Pep-FITC sensors after adding the mixture of MMP2 $(1.4 \mathrm{nM})$ and $10 \mu \mathrm{M} 1,10$-phenanthroline, a broad-spectrum inhibitor of MMPs, was largely abolished (Figure 5, curve d compared with $b$ ), indicating that the MMPs inhibitor inhibited MMP2 activation largely. We further assessed the effect of the inhibitor on the fluorescence intensity, and found that there was hardly any change in the fluorescence intensity of c-nGO/Pep-FITC (Figure 5, curve a and c) or free Pep-FITC (Figure 6) before and after adding the inhibitor, suggesting that the inhibitor had no direct effect on the fluorescence intensity of the sensor or free FITC. These data demonstrated that fluorescence recovery of the c-nGO/Pep-FITC sensor in the presence of MMP2 was due to the cleavage of the peptide by the activated MMP2.

Before we applied the $\mathrm{nrGO} / \mathrm{Pep}-\mathrm{FITC}$ sensor to detect the protease activities of MMP2, the reaction time for the detecting system was optimized. After adding MMP2 $(0.28 \mathrm{nM})$, the fluorescence intensity of $\mathrm{nrGO} / \mathrm{Pep}-\mathrm{FITC}$ sensor $(100 \mathrm{nM})$ increased gradually along with the cleavage time until it reached a plateau at 2 hours under $37^{\circ} \mathrm{C}$ in TCNB buffer (Figure 7). We thus chose 2 hours for the following detection.

After mixing $100 \mathrm{nM} \mathrm{nrGO} / \mathrm{Pep}-\mathrm{FITC}$ with MMP2 at $37^{\circ} \mathrm{C}$ for 2 hours with gentle shaking, we detected the enhanced fluorescence intensity $\Delta F\left(\Delta F=F-F_{0}\right.$, where $F$ and $F_{0}$ stand for the fluorescence intensity of nrGO/Pep-FITC sensor after and before reacting with MMP2, respectively) of $\mathrm{nrGO}$ / Pep-FITC sensor in the presence of various concentrations $(0-1.4 \mathrm{nM})$ of MMP2. As shown in Figure 8A, the fluores-
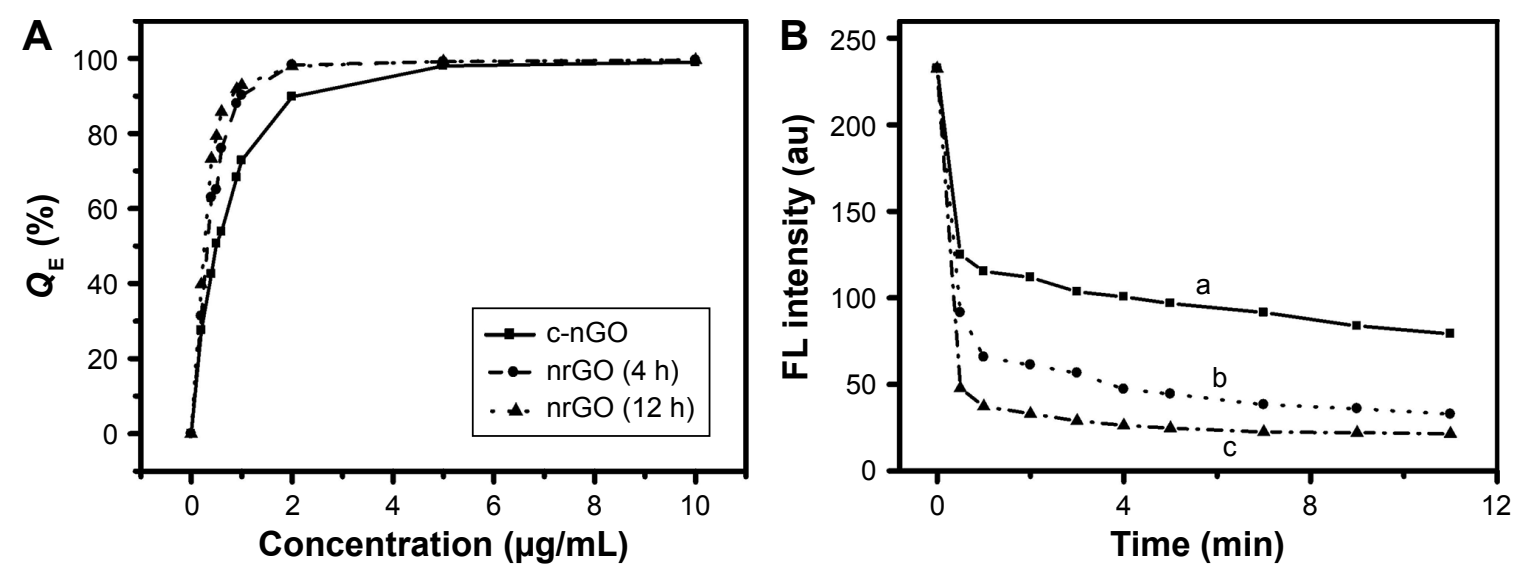

Figure 4 Quenching effect of nano-materials.

Notes: Dose-dependent quenching efficiencies $\left(Q_{E}\right)(\mathbf{A})$ of c-nGO, nrGO (4 h), and nrGO (I2 h) on Pep-FITC (I00 nM) and time-dependent fluorescence quenching (B) of Pep-FITC (100 nM) in TCNB buffer with I $\mu \mathrm{g} / \mathrm{mL}$ c-nGO (a), nrGO (4 h) (b), and nrGO (12 h) (c).

Abbreviations: c-nGO, carboxylated nano-graphene oxide; nrGO, reduced nano-graphene oxide; Pep-FITC, fluorescein isothiocyanate-labeled peptide; TCNB, Tris (50 mM), $\mathrm{CaCl}_{2}(10 \mathrm{mM}), \mathrm{NaCl}$ (I50 mM), and Brij 35 (0.05\%) (pH 7.5); FL, fluorescence; au, arbitrary unit; min, minutes; h, hours. 


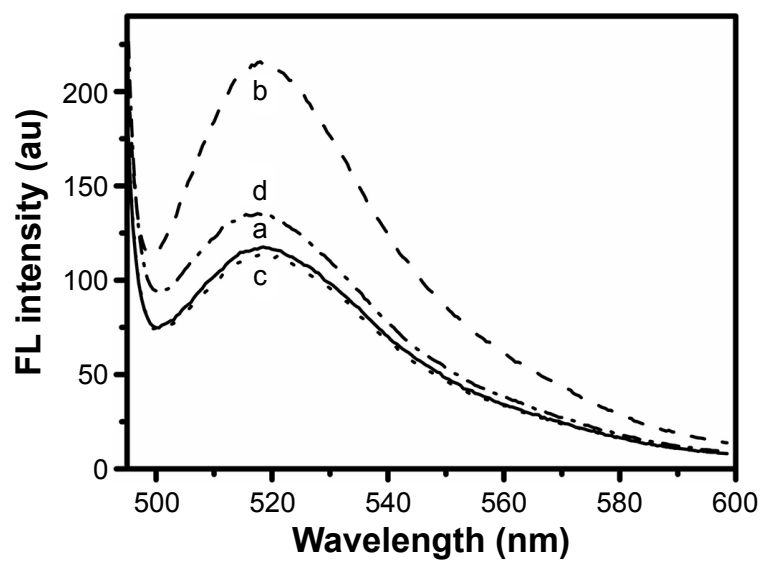

Figure 5 Fluorescence spectra of different reaction systems. Notes: c-nGO/Pep-FITC (I00 nM) (a); (a) + MMP2 (I.4nM) (b); (a) + I, I0-phenanthroline $(10 \mu M)(c)$, and $(a)+I, 10-$ phenanthroline $(10 \mu M)+$ MMP2 (I.4 nM) (d).

Abbreviations: c-nGO, carboxylated nano-graphene oxide; Pep-FITC, fluorescein isothiocyanate-labeled peptide; MMP2, matrix metalloproteinase 2; FL, fluorescence; au, arbitrary unit.

cence recovery of FITC was dependent on the concentration of MMP2 introduced. Moreover, the enhanced fluorescence intensity $\Delta F$ was linearly correlated with the MMP2 concentration in the range of 0.02-0.1 nM (inset in Figure 8A). The detection limit ( $3 S / m$, in which $S$ is the standard deviation of blank measurements, $n=10$, and $m$ is the slope of the linear equation $)^{24}$ of the nrGO/Pep-FITC was determined to be $3 \mathrm{pM}$, which is approximately tenfold lower than that of the c-nGO/Pep-FITC probe (Figure 8B), attributing to the higher ability of nrGO to absorb visible light (Figure 3C). The $3 \mathrm{pM}$ detection limit of nrGO/Pep-FITC for MMP2 is at least elevenfold lower than that of the GO-Pep-FITC sensor

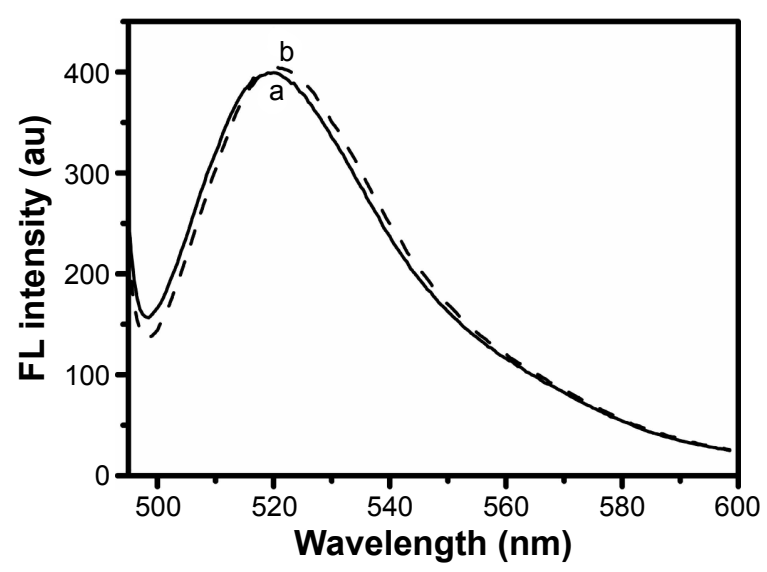

Figure 6 Effect of additional MMP2 inhibitor (1,10-phenanthroline) on the FITC fluorescence intensity.

Notes: Pep-FITC (I00 nM) (a) and Pep-FITC (100 nM) +I,I0-phenanthroline $(10 \mu \mathrm{M})(\mathrm{b})$.

Abbreviations: MMP2, matrix metalloproteinase 2; FITC, fluorescein isothiocyanate; Pep-FITC, fluorescein isothiocyanate-labeled peptide; FL, fluorescence; au, arbitrary unit.

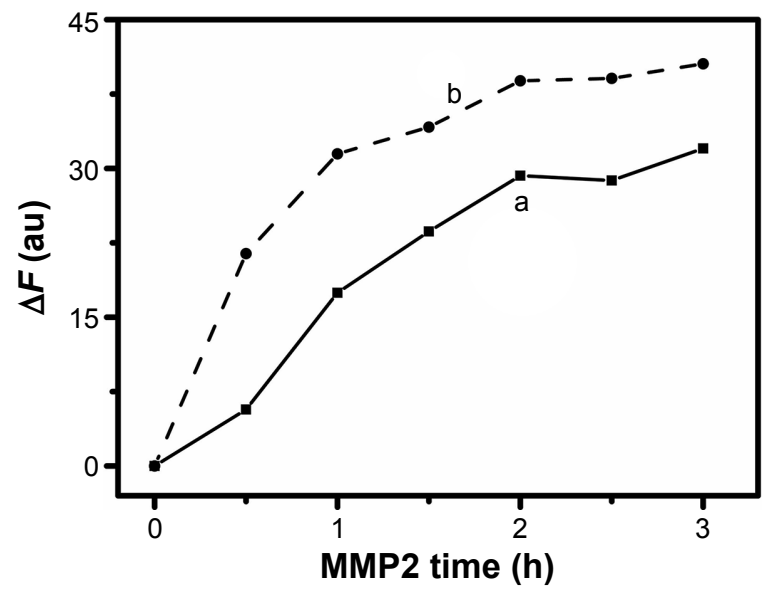

Figure 7 Time optimization of probes for MMP2 detection.

Notes: Effect of reaction time on the fluorescence change of the c-nGO/Pep-FITC (a) and nrGO/Pep-FITC (b) probes (100 nM) reacting with MMP2 (0.28 nM) in TCNB buffer at $37^{\circ} \mathrm{C}$.

Abbreviations: c-nGO, carboxylated nano-graphene oxide; Pep-FITC, fluorescein isothiocyanate-labeled peptide; nrGO, reduced nano-graphene oxide; MMP2, matrix metalloproteinase 2; TCNB, Tris (50 mM), $\mathrm{CaCl}_{2}(10 \mathrm{mM}), \mathrm{NaCl}(150 \mathrm{mM})$, and $\mathrm{Brij} 35$ $(0.05 \%)(\mathrm{pH} 7.5) ; \Delta F$, changeable fluorescence intensity; au, arbitrary unit; $\mathrm{h}$, hours.

developed by Song et $\mathrm{al}^{25}$ as well as 16 -fold lower than that of the GO-peptide sensor developed by Feng et al. ${ }^{24}$

To test the selectivity, the reaction of the nrGO/Pep-FITC $(100 \mathrm{nM})$ with $\mathrm{KCl}(150 \mathrm{mM}), \mathrm{MgCl}_{2}(2.5 \mathrm{mM}), \mathrm{ZnCl}_{2}$ (2.5 $\mathrm{mM})$, glucose $(10 \mathrm{mM})$, glycine $(1 \mathrm{mM})$, glutamine $(1 \mathrm{mM})$, bovine serum albumin $(100 \mathrm{nM})$, human serum albumin (100 nM), MMM9 (another member of MMPs, $2 \mathrm{nM})$, and MMP2 (2 $\mathrm{nM}$ ) was examined in parallel under the same conditions (incubated at $37^{\circ} \mathrm{C}$ for 2 hours). As shown in Figure 9, the nrGO/Pep-FITC sensor exhibited the strongest fluorescence response to $2 \mathrm{nM}$ of MMP2 and very weak fluorescence response to $2 \mathrm{nM}$ of MMP9, and did not show obvious response to $\mathrm{KCl}, \mathrm{MgCl}_{2}, \mathrm{ZnCl}_{2}$, glucose, glycine, glutamine, bovine serum albumin, and human serum albumin even with micromole-level concentrations. These data clearly indicate that the nrGO/Pep-FITC sensor possesses a high specificity for MMP2, which is ascribed to the cleavage of the specific substrate by MMP2.

Based on the powerful light quenching of rGO, we here devoted ourselves to develop the nrGO/Pep-FITC sensor for rapid and ultrasensitive measurement of MMP2. In fact, the nrGO/Pep-FITC sensor can also be used to monitor the trace amount of other proteases and protease inhibitors by changing polypeptide chain. For example, the expression of MMP10 in healthy human plasma is commonly $\sim 0.03 \mathrm{nM},{ }^{13}$ and the levels of MMPs in urine and saliva are much lower than that in plasma. ${ }^{48,49} \mathrm{We}$ can replace the MMP2 cleavage substrate in the Pep-FITC with MMP10 cleavage substrate for the sensitive MMP10 detection. In reality, 

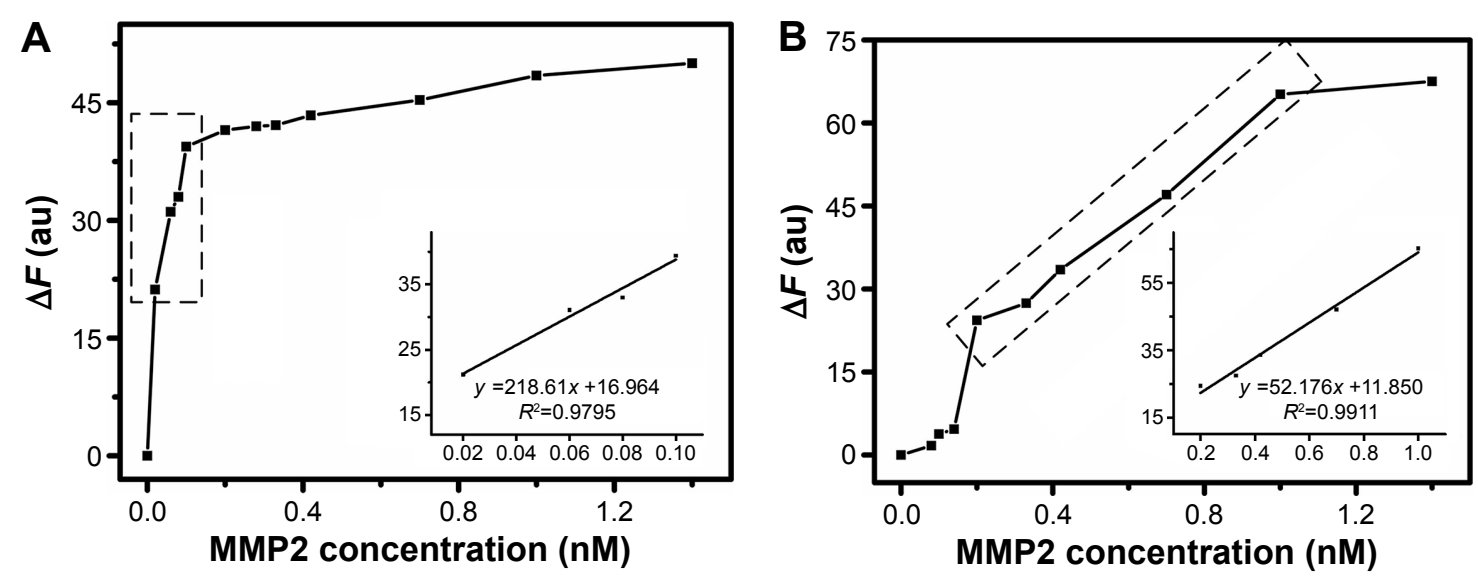

Figure 8 Linear concentration range of probes for MMP2 detection.

Notes: The changed fluorescence intensity of nrGO/Pep-FITC (A) and c-nGO/Pep-FITC (B) probes (I00 nM) versus MMP2 concentration. The insets at the bottom right indicate the linear regression of the enhanced fluorescence intensity $(\Delta F$, at $518 \mathrm{~nm})$ versus the concentration of MMP2. Linear detection range is indicated by dotted rectangle. Abbreviations: nrGO, reduced nano-graphene oxide; Pep-FITC, fluorescein isothiocyanate-labeled peptide; c-nGO, carboxylated nano-graphene oxide; MMP2, matrix metalloproteinase $2 ; \Delta F$, changeable fluorescence intensity; au, arbitrary unit.

the nrGO/Pep-FITC sensor tends to be destroyed when other similar molecules competitively bind with nrGO, limiting its practical application in complex biological samples, which is a key issue to be overcome in the near future.

\section{Conclusion}

In summary, a novel nrGO/Pep-FITC sensor for sensitive, rapid, and accurate analysis of a protease biomarker (MMP2) has been developed through non-covalent binding of PepFITC to nrGO. Compared with the unreduced c-nGO/PepFITC, nrGO/Pep-FITC developed here has lower background

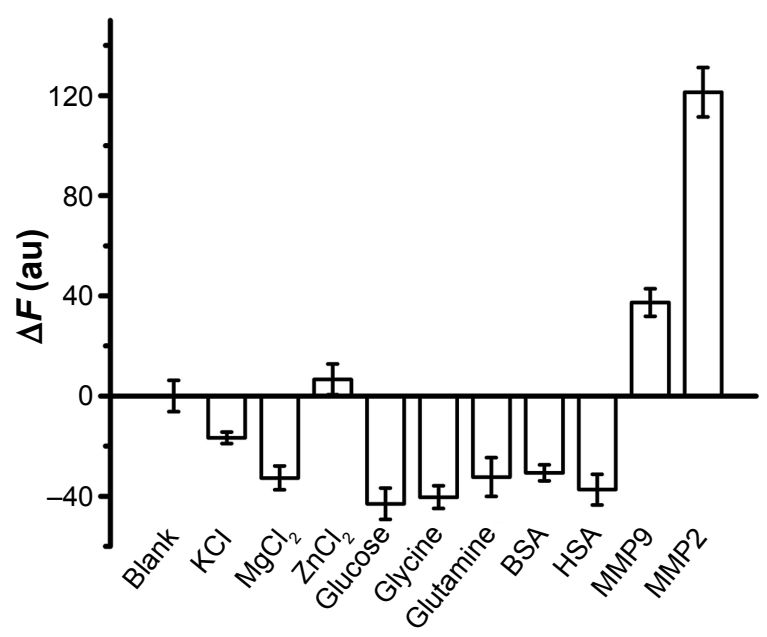

Figure 9 Fluorescence responses of the nrGO/Pep-FITC (I00 nM) to various substances: $\mathrm{KCl}(150 \mathrm{mM}), \mathrm{MgCl}_{2}(2.5 \mathrm{mM}), \mathrm{ZnCl}_{2}(2.5 \mathrm{mM})$, glucose $(10 \mathrm{mM})$, glycine (I mM), glutamine (I mM), BSA (100 nM), HSA (100 nM), MMP9 (2 nM), and MMP2 (2 nM).

Notes: $\Delta F$ is the difference of the fluorescence intensity of nrGO/Pep-FITC in the presence and absence of a substance.

Abbreviations: nrGO, reduced nano-graphene oxide; Pep-FITC, fluorescein isothiocyanate-labeled peptide; BSA, bovine serum albumin; HSA, human serum albumin; MMP9, matrix metalloproteinase 9; MMP2, matrix metalloproteinase 2. signals and a tenfold lower detection limit for MMP2. Attributed to the simple preparation and facile manipulation, we envision that this work may inspire many people to develop multifunctional rGO-based biosensor platforms for the ultrasensitive detection of trace elements by functionalizing rGO with many kinds of functional probes and expand the application fields of rGO-based sensor.

\section{Acknowledgments}

This work was supported by the National Natural Science Foundation of China (Nos 61527825, 81471699, and 61178078) and the Guangdong Province Science and Technology Plan Project (2014B090901060) as well as the Guangzhou Science and Technology Plan Project (No 2014J4100055).

\section{Author contributions}

Tongsheng Chen and Xiaoping Wang contributed to conception and study design. Gaina Xi was involved in data acquisition. Gaina $\mathrm{Xi}$ and Tongsheng Chen were involved in data analysis and interpretation. Gaina Xi performed the statistical analyses. Gaina Xi and Tongsheng Chen drafted the article. All of the authors contributed to writing and revising the manuscript for scientific content, approved the final version before submission and agree to be accountable for all aspects of the work.

\section{Disclosure}

The authors report no conflicts of interest in this work.

\section{References}

1. Chau Y, Padera RF, Dang NM, Langer R. Antitumor efficacy of a novel polymer-peptide-drug conjugate in human tumor xenograft models. Int J Cancer. 2006;118(6):1519-1526. 
2. Sternlicht MD, Werb Z. How matrix metalloproteinases regulate cell behavior. Annu Rev Cell Dev Biol. 2001;17:463-516.

3. Mitsiades N, Yu WH, Poulaki V, Tsokos M, Stamenkovic I. Matrix metalloproteinase-7-mediated cleavage of Fas ligand protects tumor cells from chemotherapeutic drug cytotoxicity. Cancer Res. 2001;61(2): $577-581$.

4. Littlepage LE, Sternlicht MD, Rougier N, et al. Matrix metalloproteinases contribute distinct roles in neuroendocrine prostate carcinogenesis, metastasis, and angiogenesis progression. Cancer Res. 2010;70(6): 2224-2234.

5. Ribatti D. Endogenous inhibitors of angiogenesis: a historical review. Leuk Res. 2009;33(5):638-644.

6. Cornelius LA, Nehring LC, Harding E, et al. Matrix metalloproteinases generate angiostatin: effects on neovascularization. J Immunol. 1998; 161(12):6845-6852.

7. Patterson BC, Sang QA. Angiostatin-converting enzyme activities of human matrilysin (MMP-7) and gelatinase B/type IV collagenase (MMP-9). J Biol Chem. 1997;272(46):28823-28825.

8. Patel S, Sumitra G, Koner BC, Saxena A. Role of serum matrix metalloproteinase-2 and -9 to predict breast cancer progression. Clin Biochem. 2011;44(10-11):869-872.

9. Incorvaia L, Badalamenti G, Rini G, et al. MMP-2, MMP-9 and activin A blood levels in patients with breast cancer or prostate cancer metastatic to the bone. Anticancer Res. 2007;27(3B):1519-1525.

10. Shpitzer T, Hamzany Y, Bahar G, et al. Salivary analysis of oral cancer biomarkers. Br J Cancer. 2009;101(7):1194-1198.

11. Smith ER, Zurakowski D, Saad A, Scott RM, Moses MA. Urinary biomarkers predict brain tumor presence and response to therapy. Clin Cancer Res. 2008;14(8):2378-2386.

12. Tayebjee MH, Lip GY, MacFadyen RJ. What role do extracellular matrix changes contribute to the cardiovascular disease burden of diabetes mellitus? Diabetic Med. 2005;22(12):1628-1635.

13. Friese RS, Rao F, Khandrika S, et al. Matrix metalloproteinases: discrete elevations in essential hypertension and hypertensive end-stage renal disease. Clin Exp Hypertens. 2009;31(7):521-533.

14. Yamane T, Mitsumata M, Yamaguchi N, et al. Laminar high shear stress upregulates type IV collagen synthesis and down-regulates MMP-2 secretion in endothelium. A quantitative analysis. Cell Tissue Res. 2010;340(3): 471-479.

15. Wang YH, Shen P, Li C, Wang YY, Liu ZH. Upconversion fluorescence resonance energy transfer based biosensor for ultrasensitive detection of matrix metalloproteinase-2 in blood. Anal Chem. 2012;84(3): $1466-1473$

16. Lakowicz JR. Principles of Fluorescence Spectroscopy. 3rd ed. Singapore: Springer; 2006:443-452.

17. Li X, Deng D, Xue JP, Qu LZ, Achilefu S, Gu YQ. Quantum dots based molecular beacons for in vitro and in vivo detection of MMP-2 on tumor. Biosens Bioelectron. 2014;61:512-518.

18. Akers WJ, Xu B, Lee H, et al. Detection of MMP-2 and MMP-9 activity in vivo with a triple-helical peptide optical probe. Bioconjug Chem. 2012;23(3):656-663.

19. Qiu HZ, Wu NM, Zheng YJ, et al. A robust and versatile signal-on fluorescence sensing strategy based on SYBR Green I dye and graphene oxide. Int J Nanomed. 2015;10:147-156.

20. He S, Song B, Li D, et al. A graphene nanoprobe for rapid, sensitive, and multicolor fluorescent DNA analysis. Adv Funct Mater. 2010;20(3): 453-459.

21. Morales-Narváez E, Merkoçi A. Graphene oxide as an optical biosensing platform. Adv Mater. 2012;24(25):3298-3308.

22. Wen J, Xu Y, Li H, Lu A, Sun S. Recent applications of carbon nanomaterials in fluorescence biosensing and bioimaging. Chem Commun. 2015; 51(57):11346-11358.

23. Li J, Lu CH, Yao QH, et al. A graphene oxide platform for energy transfer-based detection of protease activity. Biosens Bioelectron. 2011; 26(9):3894-3899.
24. Feng D, Zhang YY, Feng TT, Shi W, Li XH, Ma HM. A graphene oxide-peptide fluorescence sensor tailor-made for simple and sensitive detection of matrix metalloproteinase 2. Chem Commun. 2011;47(38): 10680-10682.

25. Song EQ, Cheng D, Song Y, Jiang MD, Yu JF, Wang YY. A graphene oxide-based FRET sensor for rapid and sensitive detection of matrix metalloproteinase 2 in human serum sample. Biosens Bioelectron. 2013;47: 445-450.

26. Kim J, Cote LJ, Kim F, Huang J. Visualizing graphene based sheets by fluorescence quenching microscopy. J Am Chem Soc. 2010;132(1): 260-267.

27. Zhu XL, Shen YL, Cao JP, et al. Detection of microRNA SNPs with ultrahigh specificity by using reduced graphene oxide-assisted rolling circle amplification. Chem Commun. 2015;51(49):10002-10005.

28. Hu H, Xin JH, Hu H, Wang X, Lu X. Organic liquids-responsive beta-cyclodextrin-functionalized graphene-based fluorescence probe: label-free selective detection of tetrahydrofuran. Molecules. 2014;19(6): 7459-7479.

29. Fan XB, Peng WC, Li Y, et al. Deoxygenation of exfoliated graphite oxide under alkaline conditions: a green route to graphene preparation. Adv Mater. 2008;20(23):4490-4493.

30. Dreyer DR, Murali S, Zhu Y, Ruoff RS, Bielawski CW. Reduction of graphite oxide using alcohols. J Mater Chem. 2011;21(10):3443-3447.

31. Wang Y, Shi ZX, Yin J. Facile synthesis of soluble graphene via a green reduction of graphene oxide in tea solution and its biocomposites. ACS Appl Mater Interfaces. 2011;3(4):1127-1133.

32. Zhou Y, Bao QL, Tang LAL, Zhong YL, Loh KP. Hydrothermal dehydration for the "green" reduction of exfoliated graphene oxide to graphene and demonstration of tunable optical limiting properties. Chem Mater. 2009;21(13):2950-2956.

33. Chen JQ, Liu HY, Zhao CB, et al. One-step reduction and PEGylation of graphene oxide for photothermally controlled drug delivery. Biomaterials. 2014;35(18):4986-4995.

34. Chen JQ, Wang XP, Chen TS. Facile and green reduction of covalently PEGylated nanographene oxide via a 'water-only' route for high-efficiency photothermal therapy. Nanoscale Res Lett. 2014;9(1):1-10.

35. Hummers Jr WS, Offeman RE. Preparation of graphitic oxide. J Am Chem Soc. 1958;80(6):1339-1339.

36. Dikin DA, Stankovich S, Zimney EJ, et al. Preparation and characterization of graphene oxide paper. Nature. 2007;448(7152):457-460.

37. Sun X, Liu Z, Welsher K, et al. Nano-graphene oxide for cellular imaging and drug delivery. Nano Res. 2008;1(3):203-212.

38. Lee S, Cha EJ, Park K, et al. A near-infrared-fluorescence-quenched gold-nanoparticle imaging probe for in vivo drug screening and protease activity determination. Angew Chem Int Ed. 2008;47: 2804-2807.

39. He HY, Klinowski J, Forster M, Lerf A. A new structural model for graphite oxide. Chem Phys Lett. 1998;287(1-2):53-56.

40. Li D, Muller MB, Gilje S, Kaner RB, Wallace GG. Processable aqueous dispersions of graphene nanosheets. Nat Nanotechnol. 2008;3(2): 101-105.

41. Luo JY, Cote LJ, Tung VC, et al. Graphene oxide nanocolloids. J Am Chem Soc. 2010;132(50):17667-17669.

42. Liu KP, Zhang JJ, Cheng FF, Zheng TT, Wang CM, Zhu JJ. Green and facile synthesis of highly biocompatible graphene nanosheets and its application for cellular imaging and drug delivery. J Mater Chem. 2011;21(32):12034-12040.

43. Cataldo F. Structural analogies and differences between graphite oxide and C-60 and C-70 polymeric oxides (fullerene ozopolymers). Fuller Nanotub Car N. 2003;11(1):1-13.

44. Hermanson GT. Bioconjugate Techniques. San Diego, CA: Academic Press; 1996:127-228.

45. Li J, Lin H, Yang Z, Li J. A method for the catalytic reduction of graphene oxide at temperatures below $150^{\circ}$ C. Carbon. 2011;49(9): 3024-3030. 
46. Zhang M, Yin BC, Wang XF, Ye BC. Interaction of peptides with graphene oxide and its application for real-time monitoring of protease activity. Chem Commun. 2011;47(8):2399-2401.

47. Wang XH, Wang CY, Qu KG, et al. Ultrasensitive and selective detection of a prognostic indicator in early-stage cancer using graphene oxide and carbon nanotubes. Adv Funct Mater. 2010;20(22):3967-3971.

48. Manicourt DH, Azria M, Mindeholm L, Thonar EJM, Devogelaer JP. Oral salmon calcitonin reduces Lequesne's algofunctional index scores and decreases urinary and serum levels of biomarkers of joint metabolism in knee osteoarthritis. Arthritis Rheum. 2006;54(10):3205-3211.
49. Asatsuma M, Ito $\mathrm{S}$, Watanabe $\mathrm{M}$, et al. Increase in the ratio of matrix metalloproteinase-9 to tissue inhibitor of metalloproteinase-1 in saliva from patients with primary Sjögren's syndrome. Clin Chim Acta. 2004;345(1):99-104.

\section{Publish your work in this journal}

The International Journal of Nanomedicine is an international, peerreviewed journal focusing on the application of nanotechnology in diagnostics, therapeutics, and drug delivery systems throughout the biomedical field. This journal is indexed on PubMed Central, MedLine, CAS, SciSearch $®$, Current Contents $® /$ Clinical Medicine,
Journal Citation Reports/Science Edition, EMBase, Scopus and the Elsevier Bibliographic databases. The manuscript management system is completely online and includes a very quick and fair peer-review system, which is all easy to use. Visit http://www.dovepress.com/ testimonials.php to read real quotes from published authors.

Submit your manuscript here: http://www.dovepress.com/international-journal-of-nanomedicine-journal 